\title{
Acupuncture for chronic neck pain: a protocol for an updated systematic review

Qinhong Zhang ${ }^{1,2+}$, Jinhuan Yue ${ }^{1+}$, Xiangxin Zeng ${ }^{2}$, Zhongren Sun ${ }^{2^{*}}$ and Brenda Golianu ${ }^{1 *}$

\begin{abstract}
Background: This study aims to investigate the efficacy and safety of acupuncture for patients with chronic neck pain. Methods: The MEDLINE, EMBASE, CENTRAL, CINAHL, and the Chinese Biomedical Literature Database, the China National Knowledge Infrastructure, VIP Information, and Wanfang Data databases will be searched from their inception to present. Randomised controlled trials (RCTs) of acupuncture (assessed as the sole treatment or as an adjunct treatment) for chronic neck pain will be included. The primary outcome is chronic neck pain measured by the visual analogue scale (VAS), McGill Pain Questionnaire, or short-form McGill Pain Questionnaire. The secondary outcomes will include the functional recovery, health-related quality of life, psychological improvements related to the reduction of pain, and adverse events. Two authors will perform the study selection, data extraction, and quality assessment independently. Any disagreements will be resolved through discussion with a third author. Methodological quality of the included trials will be evaluated by the Cochrane risk-of-bias criteria, and the Standards for Reporting Interventions in Controlled Trials of Acupuncture checklist will be used to assess completeness of reporting.
\end{abstract}

Discussion: The results of this systematic review will provide the latest evidence of the efficacy of acupuncture in treating chronic neck pain, which will benefit both practitioners and policymakers.

Systematic review registration: PROSPERO CRD42015017178

Keywords: Chronic neck pain, Acupuncture, Systematic review, Randomised controlled trial

\section{Introduction}

Neck pain is a common medical condition and a common cause of disability $[1,2]$. It is reported that a 12 month prevalence of neck pain is $30-50 \%$ in the adult population [3]. Additionally, $23 \%$ of individuals will develop a recurrent episode in the months after their recovery [4], and women are more likely than men to develop neck pain [4].

Multiple physical interventions are available to treat chronic neck pain, such as exercise $[5,6]$, traction $[7,8]$, physical therapy [9], manual therapy [10, 11], massage, and others [12]. However, a previous study, based on a rigorous assessment of randomised controlled trials (RCTs), found no clear evidence that any type of physical

\footnotetext{
* Correspondence: sunzhongren2011@163.com; bgolianu@stanford.edu ${ }^{\dagger}$ Equal contributors

2Department of Acupuncture and Moxibustion, College of Acupuncture and Moxibustion, Heilongjiang University of Chinese Medicine, Harbin 150040, China

${ }^{1}$ Department of Anesthesiology, Stanford University, 300 Pasteur Dr, Stanford, CA 94305, USA
}

therapy was more efficient than any other for chronic neck pain [13].

Acupuncture, a physical intervention which involves placement of small needles in the skin at different acupoints, has been practiced in China for 2000 years and is commonly used for many types of chronic pain [14-18]. It is believed that acupuncture relieves pain by preventing or modifying peripheral, spinal, and supraspinal mechaisms [19]. The efficacy of acupuncture for neck pain has evaluated in three systematic reviews [18, 20, 21] Alhough previous systematic reviews of neck pain have some insight into the potential benefit of previous sysreview and to further specifically and critically chronic neck pain. 


\section{Objectives}

We will conduct a systematic review to critically assess the efficacy and safety of recent clinical evidence of acupuncture for chronic neck pain.

\section{Methods}

\section{Study registration}

The protocol for this systematic review has been registered with PROSPERO 2015 (http://www.crd.york.ac.uk/ PROSPERO) under registration number CRD4201501 7178. This protocol is performed and reported according to the Preferred Reporting Items for Systematic Reviews and Meta-Analyses Protocols (PRISMA-P) statement guidelines (see Additional file 1) [22]. However, the review will be conducted depending on the Preferred Reporting Items for Systematic Reviews and MetaAnalyses (PRISMA) statement guidelines [23].

\section{Criteria for study inclusion Study types}

RCTs which compare acupuncture with sham acupuncture or other interventions in patients with chronic neck pain will be included. Non-randomised studies will be excluded. No language restriction will be applied in this study.

\section{Participants}

Studies evaluating patients diagnosed with chronic neck pain for at least 3 months will be included regardless of their age, sex, or ethnicity.

In addition, studies of chronic neck pain related to the following diagnostic categories will be included: mechanical neck disorders, including whiplash-associated disorders categories 1 and 2 [24, 25], myofascial chronic neck pain, and degenerative changes [26] and neck disorder with radicular symptoms, including whiplash-associated disorder category III, but without headache [24, 25]. Furthermore, if the participants have other types of pain in addition to chronic neck pain, we will only focus on the chronic neck pain. However, studies of chronic neck pain related to fractures and dislocations, coexisting headache or headache; other pathological entities; and definite or possible long tract signs (e.g. myelopathies) will be excluded.

\section{Interventions}

Acupuncture therapy involving body, scalp, auricular acupuncture, and electroacupuncture will be included. However, studies comparing different types of acupuncture or different points will be excluded.

Comparison interventions may be sham acupuncture (including minimal acupuncture, using of invalid points, using invalid stimulation but on appropriate location or depth, and non-penetrating sham device such as Park's sham device or mock electrical stimulation) or other therapies including no treatment, usual care, and other conventional treatments. In addition, we will include studies assessing acupuncture combined with another non-acupuncture intervention compared with the nonacupuncture intervention alone.

\section{Outcome measures}

Primary outcomes Chronic neck pain will be assessed by the visual analogue scale (VAS) (0-100) [27], McGill Pain Questionnaire (MPQ), or short-form McGill Pain Questionnaire (SF-MPQ) [28-30].

Secondary outcomes Secondary outcomes will include functional recovery (such as disability, return to activities, work, or school), health-related quality of life [31], and psychological improvements related to the reduction of pain. Additionally, side effects related to the acupuncture intervention will also be documented.

\section{Search methods to identify studies}

The search strategy is developed with the help of experienced librarians to retrieve MEDLINE, EMBASE, CENTRAL, CINAHL, the Chinese Biomedical Literature Database (CBM), the China National Knowledge Infrastructure (CNKI), VIP Information (VIP), and Wanfang Data (WANFANG) for key terms from their inception to present. We will use the following search terms: pain, neck pain, acupuncture, acupuncture therapy, manual acupuncture, electroacupuncture, scalp acupuncture, controlled trial, and randomised controlled trial. Chinese translation of the same search terms will be used in the Chinese databases. The search strategy for CENTRAL is shown in Table 1. In addition, the reference lists of

Table 1 Search strategy used in CENTRAL database

\begin{tabular}{|c|c|}
\hline Number & Search terms \\
\hline 1 & MeSH descriptor: [chronic neck pain] explode all trees \\
\hline 2 & $\begin{array}{l}\text { ((neck muscles) or (cervical plexus) or (cervical vertebrae) or } \\
\text { (Atlanto-Axial Joint) or (atlanto-occipital joint) or (spinal nerve } \\
\text { roots) or (brachial plexus)):ti, ab, kw }\end{array}$ \\
\hline 3 & or $1-2$ \\
\hline 4 & MeSH descriptor: [acupuncture] explode all trees \\
\hline 5 & MeSH descriptor: [acupuncture therapy] explode all trees \\
\hline 6 & $\begin{array}{l}\text { ((manual acupuncture) or (manual next acupuncture*) or } \\
{\text { (electroacupuncture) or (electro next acupuncture }{ }^{*} \text { or (scalp }}^{\left.\text {acupuncture }{ }^{*} \text { or (scalp next acupuncture* }\right) \text { :ti, ab, kw }}\end{array}$ \\
\hline 7 & or $4-6$ \\
\hline 8 & $\begin{array}{l}\text { MeSH descriptor: [randomized controlled trial] explode } \\
\text { all trees }\end{array}$ \\
\hline 9 & $\begin{array}{l}((\text { random) or (clinical trial*) or (clinical next trial*) or (controlled } \\
\text { clinical trial) or (controlled next clinical trial*)):ti, ab, kw }\end{array}$ \\
\hline 10 & or $8-9$ \\
\hline 11 & 3 and 7 and 10 \\
\hline
\end{tabular}

This search strategy will be modified as required for other electronic databases 
previously published reviews related to acupuncture and chronic neck pain will be searched.

\section{Data collection and analysis Study selection}

All studies will be screened based on their titles and abstracts first by two review authors (X.X.Z. and J.H.Y.) independently. After that, the full text will be reviewed and eligible studies will be selected. Potential disagreements will be resolved by discussion with a third review author (Q.H.Z.). The whole process of study selection is summarised in the PRISMA flow diagram (Fig. 1).

\section{Data extraction}

Data are extracted from included trials by two authors (J.H.Y. and Q.H.Z.) independently according to a predefined data extraction sheet. The extracted data will include the author, title, publication year, journal, location, participants, study size, randomisation, allocation concealment, blinding, interventions (acupuncture and control group), main outcomes, duration, follow-up, adverse events, withdrawals, and conflicts of interest. Reporting will be assessed for completion by utilising the Standards for Reporting Interventions in Controlled Trials of Acupuncture (STRICTA) checklist [32].

\section{Quality assessment}

Assessment of risk of bias in included studies The Cochrane risk of bias tool [33] and the STRICTA checklist for reporting intervention details of acupuncture will be used to evaluate the risk of bias and completeness of reporting of acupuncture intervention, respectively. All evaluations will be performed by two independent reviewers (Y.J.H. and Z.Q.H.). All discrepancies will be resolved by discussion with a third author (G. B. or S.Z.R.).

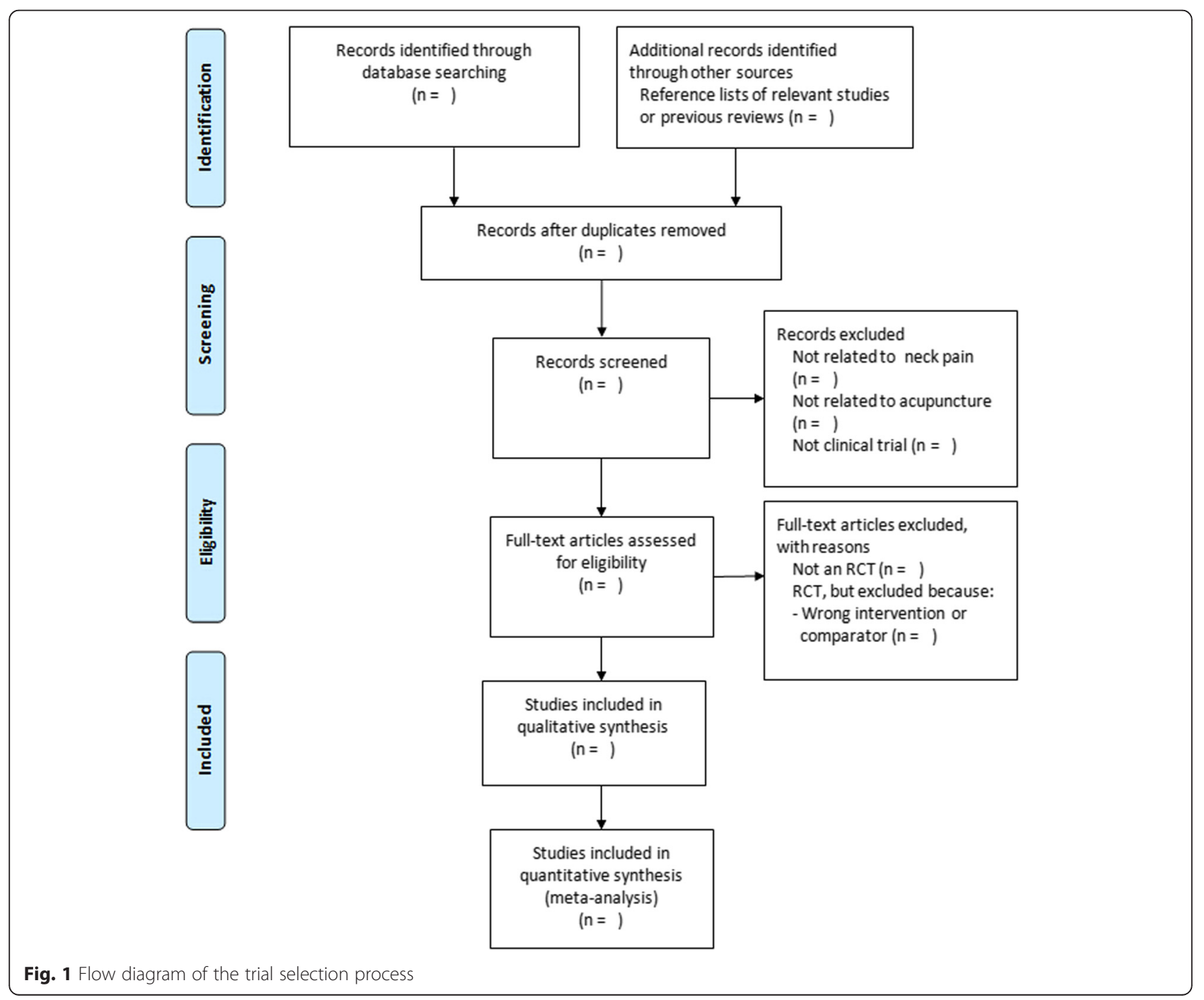


Measures of treatment effect For continuous outcomes, such as VAS or MPQ, the mean difference (MD) with a $95 \%$ confidence intervals (CIs) will be used. For dichotomous data (e.g. adverse events), risk ratio (RR) with $95 \%$ CIs will be used. Other forms of continuous or dichotomous data will be converted into MD or RR values, respectively.

\section{Missing data}

We will attempt to acquire any missing data by contacting the original study authors whenever possible. If it is not possible to get the missing data, then only the available data will be analysed.

\section{Assessment of heterogeneity}

We will evaluate heterogeneity according to the description in Section 9 of the Cochrane Handbook [34]. If significant heterogeneity exists, we will perform a subgroup analysis to explore the possible causes [34].

\section{Assessment of reporting biases}

We will use funnel plots to detect potential reporting biases. It will be used to analyse the asymmetry if at least ten trials are available in the meta-analysis [35].

\section{Data synthesis}

If it is possible to conduct a meta-analysis, Review Manager (version 5.3) software [36] (the Cochrane Collaboration, Oxford, England) will be used to combine the RR for dichotomous outcomes and MD for continuous data both with $95 \%$ CIs. Random effects model will be used if high level of clinical heterogeneity is expected due to a diverse style of acupuncture practice, different non-clinical backgrounds (country, culture, or healthcare systems) and trial settings. Otherwise, we will apply a fixed effects model. If any meta-analysis cannot be performed, we will report the results as the narrative description.

\section{Subgroup analysis}

Subgroup analysis will be conducted to assess the heterogeneity between the included trials. The analysis will include the type of acupuncture and control, such as acupuncture versus sham acupuncture (including nonpenetrating acupuncture) and acupuncture versus active comparator.

\section{Sensitivity analysis}

Sensitivity analysis will be conducted by excluding the included studies at high risk of bias for any one or more of selection, attrition, or detection bias. The metaanalysis will be repeated after removing the lower quality trials. The results will be compared and discussed according to the pooled effect size.

\section{Discussion}

Acupuncture utilisation continues to increase, with over 3 million adults undergoing acupuncture every year, often for chronic pain [37, 38]. Initial analyses suggest that acupuncture may also be a cost-effective intervention in the management of a number of painful conditions, including headache, neck pain, and back pain [39-41]. The evidence base to guide its rational use needs to be continually updated and revised to update standards of care.

Three previous systematic reviews have analysed randomised controlled trial acupuncture treatment of neck pain $[18,20,21]$. While some studies have excellent methodology, the heterogeneity of the study protocols prevented them from being able to draw firm conclusions about the efficacy of acupuncture. Thus, a new updated, comprehensive, and objective systematic for the clinical efficacy and safety of acupuncture for chronic neck pain would be of benefit to provide the needed evidence base for further treatment recommendations. This systematic review will provide a detailed summary and latest analysis of the current evidence for the efficacy of acupuncture in treating chronic neck pain, which will inform patient care, as well as health policy.

\section{Stage of review at PROSPERO}

The stage of review at PROSPERO is data extraction.

\section{Additional files}

Additional file 1: PRISMA-P checklist. PRISMA-P (Preferred Reporting Items for Systematic review and Meta-Analysis Protocols) 2015 checklist: recommended items to address in a systematic review protocol. (DOC $90 \mathrm{~kb}$ )

\begin{abstract}
Abbreviations
CBM: Chinese Biomedical Literature Database; Cls: confidence intervals; CNKI: China National Knowledge Infrastructure Database; MD: mean difference; MPQ: McGill Pain Questionnaire; PRISMA: Preferred Reporting Items for Systematic Reviews and Meta-Analyses; PRISMA-P: Preferred Reporting Items for Systematic Reviews and Meta-Analyses Protocol; RCTs: randomised controlled trials; RR: risk ratio; SF-MPQ: short-form McGill Pain Questionnaire; STRICTA: Standards for Reporting Interventions in Controlled Trials of Acupuncture; VAS: visual analogue scale; VIP: VIP Information; WANFANG: Wanfang Data.
\end{abstract}

Competing interests

The authors declare that they have no competing interests.

\begin{abstract}
Authors' contributions
$J H Y$ and $\mathrm{QHZ}$ contributed equally to this work. JHY will select the studies, extract the data, assess the risk of bias, and design the study. XXZ will select the studies and extract the data. QHZ will select the studies, extract the data, assess the risk of bias, and design the study. ZRS will assess the risk of bias. BG will assess the risk of bias and design the study. All authors contributed to the further writing of the manuscript as well as read and approved the final manuscript.
\end{abstract}

Received: 11 February 2016 Accepted: 26 April 2016 Published online: 04 May 2016 


\section{References}

1. Fejer R, Kyvik KO, Hartvigsen J. The prevalence of neck pain in the world population: a systematic critical review of the literature. Eur Spine J. 2006; 15(6):834-48.

2. Haldeman S, Carroll L, Cassidy JD, et al. The bone and joint decade 20002010 task force on neck pain and its associated disorders: executive summary. J Manipulative Physiol Ther. 2008;32 Suppl 2:S7-9.

3. Hogg-Johnson S, van der Velde G, Carroll LJ, et al. The burden and determinants of neck pain in the general population: results of the bone and joint decade 2000-2010 task force on neck pain and its associated disorders. Spine (Phila Pa 1976). 2008;33(4 Suppl):S39-51.

4. Côté P, Cassidy JD, Carroll LJ, et al. The annual incidence and course of neck pain in the general population: a population based cohort study. Pain. 2004;112:267-73

5. Yamato TP, Saragiotto BT, Maher C. Therapeutic exercise for chronic nonspecific neck pain. Br J Sports Med. 2014; bjsports-2014-093874.

6. Maiers M, Bronfort G, Evans R, et al. Spinal manipulative therapy and exercise for seniors with chronic neck pain. Spine J. 2014;14(9):1879-89.

7. Chiu TT, Ng JK, Walther-Zhang B, et al. A randomized controlled trial on the efficacy of intermittent cervical traction for patients with chronic neck pain. Clin Rehabil. 2011;25(9):814-22.

8. Borman $\mathrm{P}$, Keskin D, Ekici B, et al. The efficacy of intermittent cervical traction in patients with chronic neck pain. Clin Rheumatol. 2008;27(10):1249-53.

9. Bruflat AK, Balter JE, McGuire D, et al. Stress management as an adjunct to physical therapy for chronic neck pain. Phys Ther. 2012:92(10):1348-59.

10. Akhter S, Khan M, Ali SS, et al. Role of manual therapy with exercise regime versus exercise regime alone in the management of non-specific chronic neck pain. Pak J Pharm Sci. 2014;27(6 Suppl):2125-8.

11. Koes BW. Manual therapy for neck pain: increasing evidence for effectiveness. Ned Tijdschr Geneeskd. 2012;156(15):A4599.

12. Sherman KJ, Cherkin DC, Hawkes RJ, et al. Randomized trial of therapeutic massage for chronic neck pain. Clin J Pain. 2009;25(3):233-8.

13. van Tulder MW, Goossens M, Hoving J. Nonsurgical treatment of chronic neck pain. In: Nachemson A, Jonsson $E_{1}$ editors. Neck and back pain Philadelphia: Lippincott Williams \& Wilkins; 2000

14. Vickers AJ, Cronin AM, Maschino AC, et al. Acupuncture for chronic pain: individual patient data meta-analysis. Arch Intern Med. 2012;172(19):1444-53.

15. Hinman RS, McCrory $P$, Pirotta $M$, et al. Acupuncture for chronic knee pain: a randomized clinical trial. JAMA. 2014;312(13):1313-22.

16. Witt $C$, Brinkhaus $B$, Jena $S$, et al. Acupuncture in patients with osteoarthritis of the knee: a randomised trial. Lancet. 2005;366(9480):136-43.

17. Vas J, Perea-Milla E, Méndez C, et al. Efficacy and safety of acupuncture for chronic uncomplicated neck pain: a randomised controlled study. Pain. 2006:126(1-3):245-55.

18. Fu LM, Li JT, Wu WS. Randomized controlled trials of acupuncture for neck pain: systematic review and meta-analysis. J Altern Complement Med. 2009; 15(2):133-45.

19. Zhang R, Lao L, Ren K, Berman BM. Mechanisms of acupunctureelectroacupuncture on persistent pain. Anesthesiology. 2014;120(2):482-503.

20. White $A R$, Ernst E. A systematic review of randomized controlled trials of acupuncture for neck pain. Rheumatology (Oxford). 1999;38(2):143-7.

21. Trinh KV, Graham N, Gross AR, et al. Acupuncture for neck disorders. Cochrane Database Syst Rev. 2006;3, CD004870.

22. Shamseer $L$, Moher $D$, Clarke $M$, et al. Preferred reporting items for systematic review and meta-analysis protocols (PRISMA-P) 2015: elaboration and explanation. BMJ. 2015;349:g7647.

23. Liberati A, Altman DG, Tetzlaff J, et al. The PRISMA statement for reporting systematic reviews and meta-analyses of studies that evaluate health care interventions: explanation and elaboration. Ann Intern Med. 2009:151:W-65-94.

24. Spitzer WO, Leblanc FE, Dupuis M. Scientific approach to the assessment and management of activity related spinal disorders. Spine. 1987;7(suppl):1-59.

25. Spitzer WO, Skovron ML, Salmi LR, et al. Scientific monograph of the Quebec Task Force on Whiplash-Associated Disorders: redefining 'whiplash' and its management. Spine. 1995;20(suppl):1-73.

26. Schumacher HR, Klippel JH, Koopman WJ, editors. Primer on the rheumatic diseases. 10th ed. Atlanta: Arthritis Foundation; 1993.

27. Crichton N. Information point: visual analogue scale (VAS). J Clin Nurs. 2001; 10:697-706.

28. Burckhardt CS. The use of the McGill Pain Questionnaire in assessing arthritis pain. Pain. 1984;19:305-14.
29. Melzack R. The McGill Pain Questionnaire: major properties and scoring methods. Pain. 1975:1:277-99.

30. Melzack R. The short-form McGill Pain Questionnaire. Pain. 1987;30:191-7.

31. Brazier JE, Harper R, Jones NM, et al. Validating the SF-36 health survey questionnaire: new outcome measure for primary care. BMJ. 1992; 305(6846):160-4

32. MacPherson $\mathrm{H}$, Altman DG, Hammerschlag $\mathrm{R}$, et al. Revised standards for reporting interventions in clinical trials of acupuncture (STRICTA): extending the CONSORT statement. J Evid Based Med. 2010:3(3):140-55.

33. Higgins JPT, Altman DG and Sterne JAC on behalf of the Cochrane Statistical Methods Group and the Cochrane Bias Methods Group (editors). Chapter 8: assessing risk of bias in included studies. In: Higgins JPT, Green S, editors. Cochrane handbook for systematic reviews of interventions version 5.1.0 [updated March 2011]. The Cochrane Collaboration, 2011: Available from www.cochrane-handbook.org (accessed 2015 February 4).

34. Deeks JJ, Higgins JPT, Altman DG. Chapter 9: analysing data and undertaking meta-analyses. In: Higgins JPT, Green S, editors. Cochrane handbook for systematic reviews of interventions version 5.1.0 (updated March 2011) The Cochrane Collaboration. http://www.cochrane-handbook.org, 2011.

35. Sterne JAC, Egger M, Moher D. Chapter 10: addressing reporting biases. In: Higgins JPT, Green S, editors. Cochrane handbook for systematic reviews of interventions version 5.1.0 (updated March 2011) The Cochrane Collaboration. http://www.cochrane-handbook.org, 2011.

36. Review Manager (RevMan) [Computer program]. Version 5.3. Copenhagen: The Nordic Cochrane Centre, The Cochrane Collaboration, 2014

37. Barnes PM, Bloom B, Nahin RL. Complementary and alternative medicine use among adults and children: United States. 2008.

38. Clarke TC, Black LI, Stussman BJ, Barnes PM, Nahin RL. Trends in the use of complementary health approaches among adults: United States. 2015.

39. Vickers AJ, Rees RW, Zollman CE, McCarney R, Smith CM, Ellis N, et al. Acupuncture of chronic headache disorders in primary care: randomized controlled trial and economic analysis. Health Technol Assess. 2004;8(48):1-35.

40. Van der Velde G, Yu H, Paulden M, Cote P, Varatharajan S, Shearer HM, et al. Which interventions are cost-effective for the management of whiplashassociated and neck pain-associated disorders? A systematic review of the health and economic literature by the Ontario Protocol for Traffic Injury Management (optima) Collaboration. Spine J. 2015:S1529-9430(15):01235-8.

41. Ratcliffe J, Thomas KJ, MacPherson H, Brazier J. A randomized controlled trial of acupuncture care for persistent low back pain: cost effectiveness analysis. BMJ. 2006:333(7569):626.

\section{Submit your next manuscript to BioMed Central and we will help you at every step:}

- We accept pre-submission inquiries

- Our selector tool helps you to find the most relevant journal

- We provide round the clock customer support

- Convenient online submission

- Thorough peer review

- Inclusion in PubMed and all major indexing services

- Maximum visibility for your research

Submit your manuscript at www.biomedcentral.com/submit
) Biomed Central 